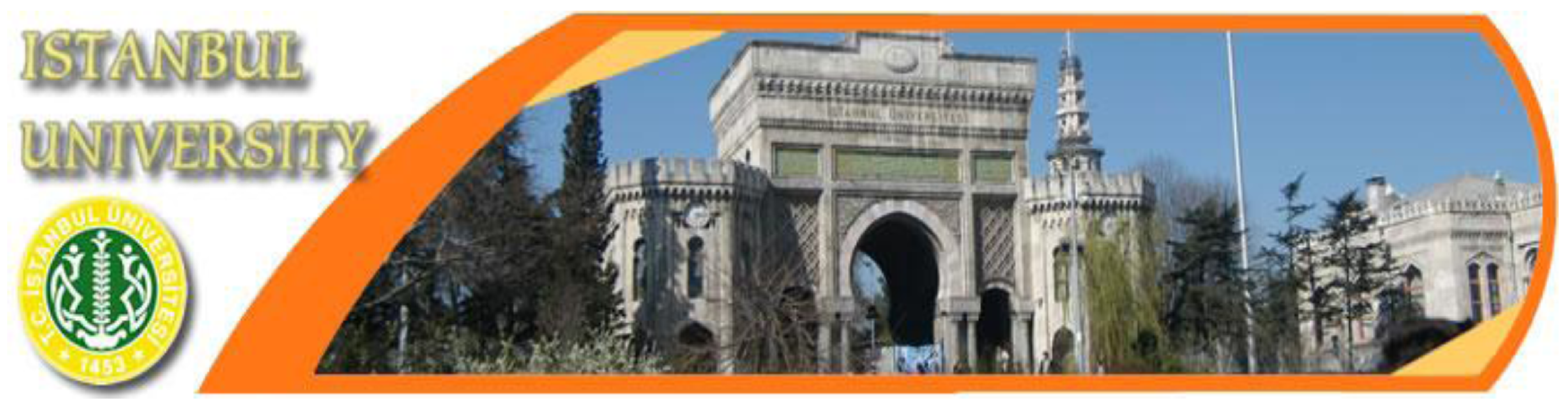

\title{
Diabetes Mellitus and Cognitive Impairment
}

\author{
Gulin Alkan¹, Seher Tanrikulu1, Sukriye Akca Kalem², Birsu Beser², Cemile Idiz', \\ Ilhan Satman ${ }^{1}$, Sibel Cakir ${ }^{2}$, Nevin Dinccag ${ }^{1}$
}

1'stanbul University, Istanbul Faculty of Medicine, Department of Internal Medicine, Division of Endocrinology and Metabolism, ${ }^{2}$ Istanbul University, Istanbul Faculty of Medicine, Department of Psychiatry, Istanbul, Turkey

Introduction: Diabetic patients have an increased risk of developing dementia and cognitive impairments. Type 2 diabetes mellitus(T2DM) related factors such as macrovascular and microvascular complications, glucose toxicity, hyperinsulinemia have been suggested to be involved cognitive impairment even in prediabetic stage. Additionally risk factors that are linked to T2DM; hypertension, obesity, dyslipidemia can also cause cognitive impairments. Our aim is to establish the relationship between different stage of T2DM and cognitive impairment.

Research design and methods: Twenty healthy controls (group 1), twenty prediabetic patients who were evaluated using a standard OGTT (group 2), fourty patients with T2DM were included in our study. Fourty patients with T2DM were divided into two groups; wellcontrolled (A1c<7.5\%) (group 3) and poorly controlled (A1c> 7.5\%) (group4) based on their HbA1c. All patients in groups were matched for sex ratio and level of education. Factors that might affect cognitive function; hypothyroidism, vitamin B12 deficiency , renal failure (GFR MDRD $<60 \mathrm{ml} / \mathrm{min}$ ), neurological and psychiatric comorbidities, any drugs that can affect cognition and also patients using insulin were evaluated and excluded from the study. We assesed neuropsychological profile of these groups and compared with group 1. Attention \& working memory, psychomotor speed, verbal memory, visuospatial memory and executive function were tested.

Results: Verbal Memory Process test score was significantly higher in group 4 than group 1 and group 2 as shown figure 1 ( $p$ value $<0.05$ ). In Trail Making Test part B diabetic patients showed significantly lower performances than group 1 and group 2 . Wisconsin Card Sorting Test (WCST) perseverative percentage was worse in prediabetic, and diabetic patients than control group which is shown in figure 2 ( $p$ value $<0.05)$

Figure 1. Verbal Memory Process Test results

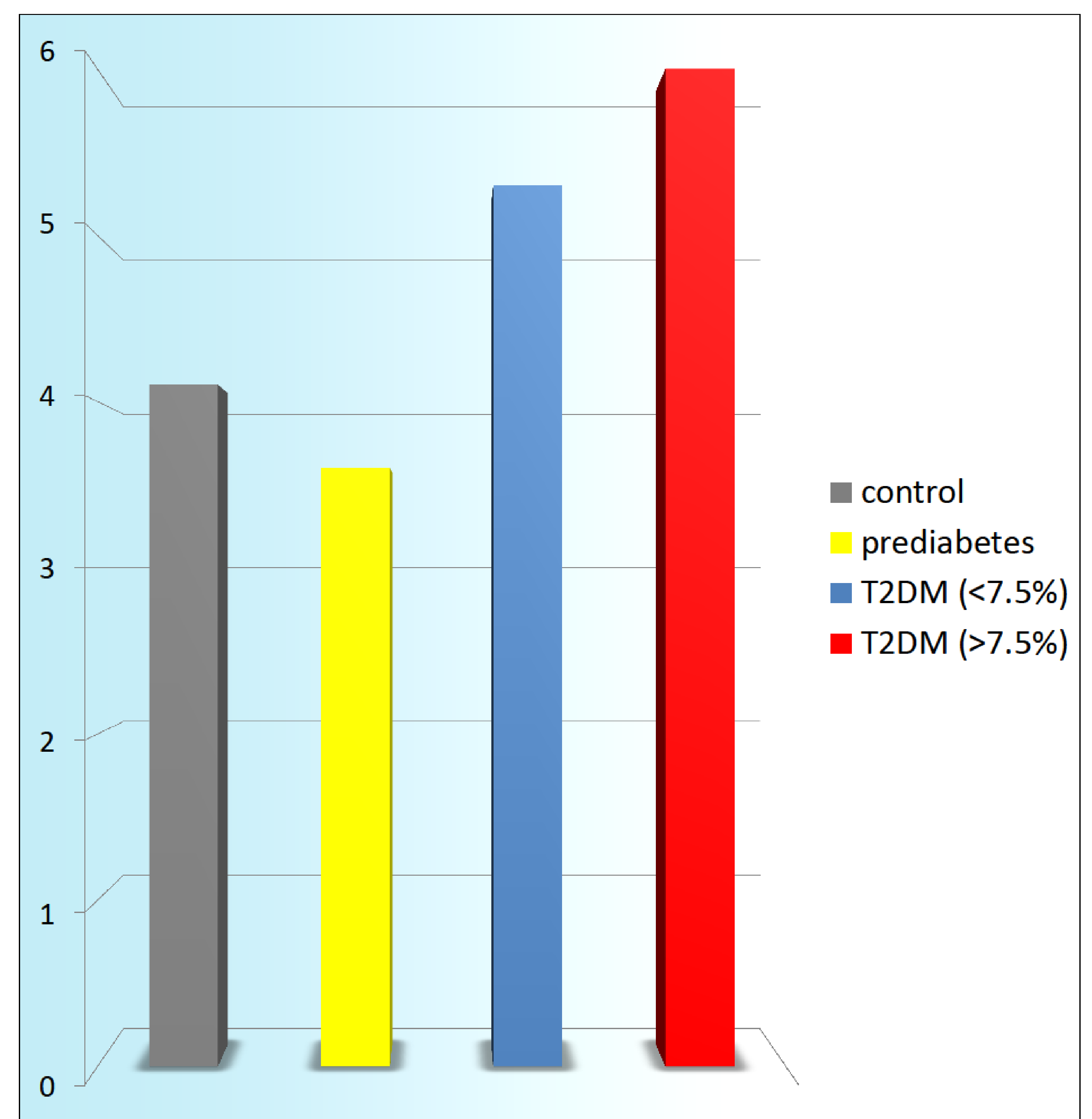

Figure 2. Wisconsin Card Sorting Test perseveration percentage

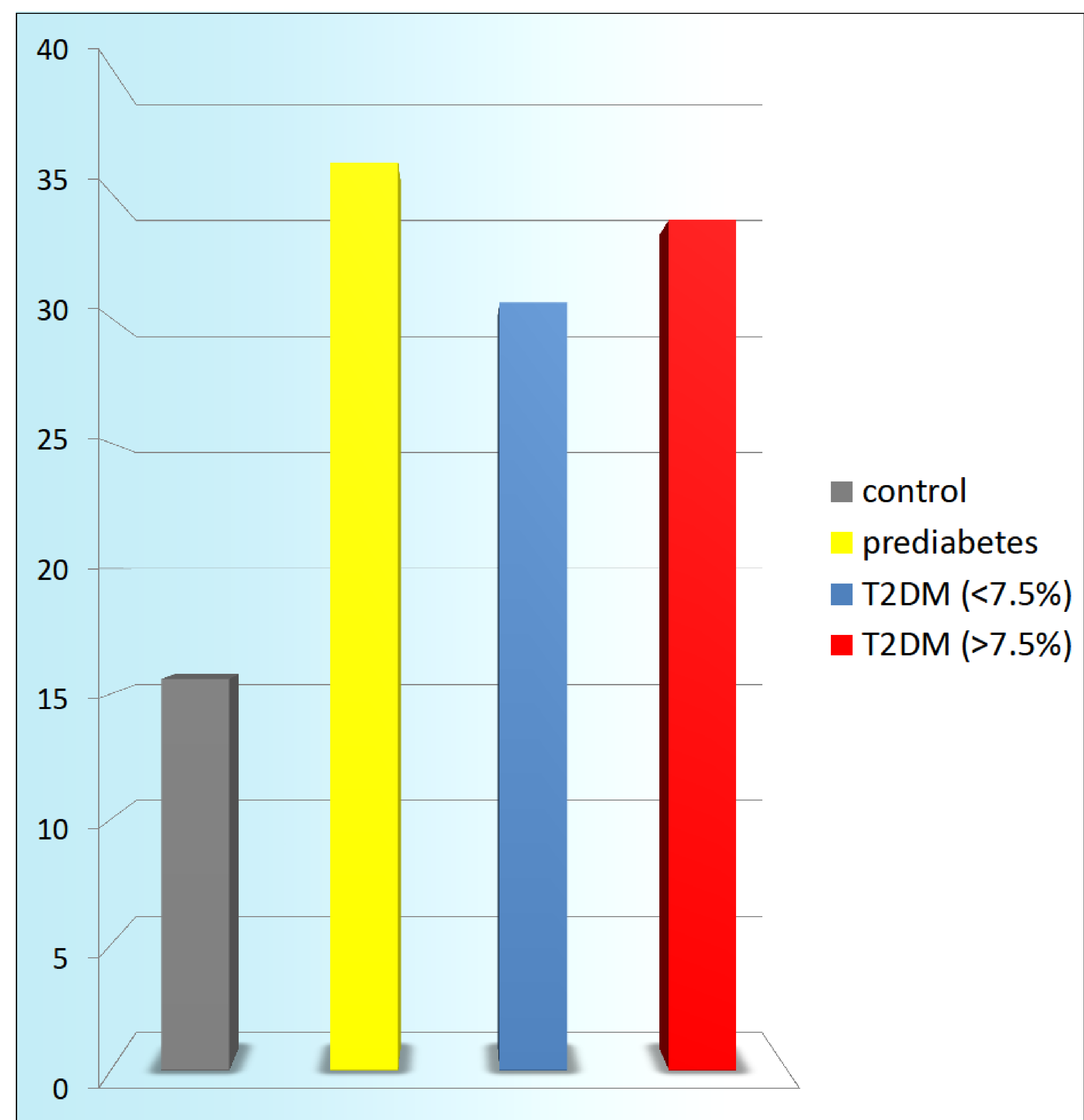

Conclusion: T2DM causes early brain ageing and declines cognitive functions even in prediabetic stage. Chronic hyperglycemia and vascular complications in poorly controlled T2DM worsen cognitive impairments. Diminished performance in executive function, verbal memory and psychomotor speed might have been more related to diabetes-related cognitive impairment.

1. Satman I, YIlmaz T, Sengul A et al. Population-based study of Diyabetes and risk characteristics in Turkey: Results of the Turkish Diabetes Epidemiology Study (TURDEP). Diabetes Care. 2002;25 $1551-1556$

2. Taylor WD, MacFall JR, Provenzale JM, Payne ME, et all. Serial MR imaging of volumes of hyperintense white matter lesions in elderly patients: correlation with vascular risk factors. AJR Am $J$ Roentgenol 2003;181:571-6.

Nothing to Disclose: GA, ST, SA, BB, CI,

IS, SC, ND 